\title{
Integrability and cosmological solutions in Einstein-æther-Weyl theory
}

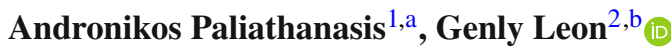 \\ ${ }^{1}$ Institute of Systems Science, Durban University of Technology, Durban 4000, South Africa \\ 2 Departamento de Matemáticas, Universidad Católica del Norte, Avda. Angamos 0610, Casilla 1280, Antofagasta, Chile
}

Received: 5 January 2021 / Accepted: 6 March 2021 / Published online: 23 March 2021

(C) The Author(s) 2021

\begin{abstract}
We consider a Lorentz violating scalar field cosmological model given by the modified Einstein-æther theory defined in Weyl integrable geometry. The existence of exact and analytic solutions is investigated for the case of a spatially flat Friedmann-Lemaître-Robertson-Walker background space. We show that the theory admits cosmological solutions of special interests. In addition, we prove that the cosmological field equations admit the Lewis invariant as a second conservation law, which indicates the integrability of the field equations.
\end{abstract}

\section{Introduction}

A plethora of modified or alternative theories to Einstein's gravity $[1,2]$ have been proposed during the last years in order to explain the cosmological observations. There is a family of theories which violate the Lorentz symmetry. The main representatives of the Lorentz violating gravitational theories are the Hořava-Lifshitz theory [3] and the Einsteinæther theory $[4,5]$.

Hořava-Lifshitz gravity is a power-counting renormalizable theory with consistent ultra-violet behaviour exhibiting an anisotropic Lifshitz scaling between time and space at the ultra-violet limit [3]. On the other hand, in Einstein-æther theory, the quadratic invariants of the kinematic quantities of a unitary time-like vector field, which is called æther field, are introduced in the gravitational Action integral; modifying the Einstein-Hilbert Action [4]. The Einstein-æther action is the most general second-order theory which is defined by the spacetime metric and the æther field involving no more than two derivatives [6] (not including total derivatives). There are several gravitational and cosmological applications for both

\footnotetext{
a e-mail: anpaliat@phys.uoa.gr

be-mail: genly.leon@ucn.cl (corresponding author)
}

of these theories in the literature, for instance see [7-24] and references therein.

Scalar fields play a significant role in the explanation of the early acceleration phase of the universe known as inflation. Lorentz violating scalar field theories have been studied in Hořava-Lifshitz gravity [25-28] and in the Einsteinæther theory [5,29-36]. In [29] it has been proposed an Einstein-æther scalar field model in which the coupling coefficients of the æther field with gravity are functions of the scalar fields. From the latter an interaction between the scalar field and the æther field it follows. For that model it was found that the inflationary era is divided into two parts, a Lorentz-violating stage and the standard slow-roll stage. In the Lorentz-violating stage the universe expands as an exact de Sitter spacetime, although the inflaton field is rolling down the potential.

In this work we are interest on the existence of exact and analytic solutions for a Lorentz-violating scalar field cosmological model. We consider the Einstein-æther theory defined in Weyl integrable geometry [37]. In this specific theory the Action Integral of the Einstein-æther is modified such that a scalar field coupled to the æther field is introduced in a geometric way. The global dynamics of the background space were studied in [37] for various cosmological models in the absence or in the presence of matter. It was found that the model provides several cosmological eras in agreement with the cosmological history. In addition, a Weyl manifold is a conformal manifold equipped with a connection which preserves the conformal structure and is torsion-free. In Weyl integrable theory the geometry is supported by the metric tensor and a connection structure which differs from the conformally equivalent metric by a scalar field [38,39]. Cosmological and gravitational applications of the Weyl geometry can be found for instance in [48-57]. The novelty of the Weyl geometry is that the scalar field in the gravitational Action integral is introduced by the geometry. 
The context of integrability is essential in all areas of physics. A set of differential equations describing a physical system is said to be integrable if there exist a sufficient number of invariant functions such that the dynamical system can be written in algebraic form. When the latter algebraic system is explicitly solved the solution of the dynamical system can be expressed in closed-form [59,60]. The study of integrability properties of nonlinear dynamical systems is important, because analytical techniques can be applied for the better understanding of the physical phenomena. Although, nowadays we have powerful computers and numerical techniques to solve nonlinear differential equations, as Arscott discussed on the preface of his book [61] "[...] fall back on numerical techniques savours somewhat of breaking a door with a hammer when one could, with a little trouble, find the key".

The plan of the paper is as follows. In Sect. 2 we present the cosmological model under consideration which is that of Einstein-æther defined in Weyl integrable geometry assuming a spatially flat Friedmann-Lemaître-Robertson-Walker (FLRW) background space without any matter source terms. In Sect. 3 we present for the first time analytic and exact solutions for this cosmological model, we focus on the existence of exact solutions where the scale factor describes inflationary models of special interests. We obtain those solutions which are determined as the general analytic solutions for the corresponding scalar field potentials. In Sect. 4 we show that this is possible because the cosmological field equations form an integrable dynamical system, where the conservation laws are the constraint cosmological equation, i.e. the modified first Friedmann's equation and the Lewis invariant. The later invariant is essential for the study of integrability of time-dependent classical or quantum systems. We show that the field equations form an integrable dynamical system for an arbitrary potential function. Finally, in Sect. 5 we summarize our results and we draw our conclusions.

\section{Einstein-æther-Weyl theory}

The Einstein-æther-Weyl gravitational model is an extension of the Lorentz violating Einstein-æther theory in Weyl integrable geometry. It is a scalar field Lorentz violating theory where there is a coupling between the scalar field and the æther field. The corresponding gravitational Action integral has the form of Einstein-æther gravity, thus it is generalized in Weyl integrable geometry. The latter generalization provides a geometric mechanism for the introduction of the scalar field into the gravitational theory.

Weyl geometry is a generalization of Riemannian geometry where the metric tensor and the covariant derivative $\left\{g_{\mu \nu}, \nabla_{\mu}\right\}$, are generalized to $\left\{\tilde{g}_{\mu \nu}, \tilde{\nabla}_{\mu}\right\}$, where $\tilde{\nabla}_{\mu}$ is not defined by the Levi-Civita connection of $g_{\mu \nu}$, but by the affine connection $\tilde{\Gamma}_{\mu \nu}^{\kappa}(\tilde{g})$ with the property [62] $\tilde{\nabla}_{\kappa} g_{\mu \nu}=\omega_{\kappa} g_{\mu \nu}$,

and $\tilde{g}_{\mu \nu}$ is the metric compatible with $\tilde{\nabla}_{\mu}$. We study Weyl integrable geometry, where the gauge vector field $\omega_{\mu}$ which defines the geometry is a gradient vector field, i.e., it satisfies $\omega_{\mu}=\phi, \mu$ for a scalar field $\phi$. Then, it is defined the new metric tensor $\tilde{g}_{\mu \nu}=e^{-\phi} g_{\mu \nu}$ as the conformally related metric compatible with the covariant derivative $\tilde{\nabla}_{\mu}$, i.e., $\tilde{\nabla}_{\kappa} \tilde{g}_{\mu \nu}=0$. Connections $\tilde{\Gamma}_{\mu \nu}^{\kappa}$ can be constructed from the Christoffel symbols $\Gamma_{\mu \nu}^{\kappa}(g)$ of the metric tensor $g_{\mu \nu}$ as follows [58]:

$\tilde{\Gamma}_{\mu \nu}^{\kappa}=\Gamma_{\mu \nu}^{\kappa}-\phi_{,(\mu} \delta_{\nu)}^{\kappa}+\frac{1}{2} \phi^{, \kappa} g_{\mu \nu}$.

The gravitational integral of the Einstein-æther-Weyl theory is [37]:

$$
\begin{aligned}
S_{A E W}\left(g_{\mu \nu}, \tilde{\Gamma}_{\mu \nu}^{\kappa} ; u^{\mu}\right)= & S_{W}\left(g_{\mu \nu}, \tilde{\Gamma}_{\mu \nu}^{\kappa}\right) \\
& +S_{A E}\left(g_{\mu \nu}, \tilde{\Gamma}_{\mu \nu}^{\kappa} ; u^{\mu}\right),
\end{aligned}
$$

where $S_{W}\left(g_{\mu \nu}, \tilde{\Gamma}_{\mu \nu}^{\kappa}\right)$ is the extension of the EinsteinHilbert action in Weyl geometry [58]:

$S_{W}\left(g_{\mu \nu}, \tilde{\Gamma}_{\mu \nu}^{\kappa}\right)=\int d x^{4} \sqrt{-g}\left(\tilde{R}+\xi\left(\tilde{\nabla}_{\nu}\left(\tilde{\nabla}_{\mu} \phi\right)\right) g^{\mu \nu}\right)$,

with $\tilde{R}$ denoting the Weylian scalar curvature

$$
\tilde{R}=R-\frac{3}{\sqrt{-g}}\left(g^{\mu \nu} \sqrt{-g} \phi\right)_{, \mu \nu}+\frac{3}{2}\left(\tilde{\nabla}_{\mu} \phi\right)\left(\tilde{\nabla}_{\nu} \phi\right)
$$

and $\xi$ is an arbitrary coupling constant. $S_{A E}\left(\tilde{g}_{\mu \nu}, \tilde{\Gamma}_{\mu \nu}^{\kappa} ; u^{\mu}\right)$ is the Action Integral for the æther field $u^{\mu}$ defined in Weyl geometry, that is:

$$
\begin{aligned}
S_{A E}\left(g_{\mu \nu}, \tilde{\Gamma}_{\mu \nu}^{\kappa} ; u^{\mu}\right)= & \int d^{4} x \sqrt{-\tilde{g}}\left(\tilde{K}^{\alpha \beta \mu \nu} \tilde{\nabla}_{\alpha} u_{\mu} \tilde{\nabla}_{\beta} u_{\nu}\right. \\
& \left.+\lambda\left(\tilde{g}_{\mu \nu} u^{\mu} u^{\nu}+1\right)\right),
\end{aligned}
$$

where $\tilde{g}_{\mu v}=e^{-\phi} g_{\mu \nu}$ is the conformally related metric associated with the covariant derivative $\tilde{\nabla}_{\mu}$.

Parameters $c_{1}, c_{2}, c_{3}$ and $c_{4}$ are dimensionless constants and define the coupling between the æether field and the conformal metric through a kinetic term. Lagrange multiplier $\lambda$ ensures the unitarity of the æther field, i.e. $\tilde{g}_{\mu \nu} u^{\mu} u^{\nu}=-1$, while the fourth-rank tensor $\tilde{K}^{\alpha \beta \mu \nu}$ is defined as

$$
\begin{aligned}
& \tilde{K}^{\alpha \beta \mu \nu} \equiv c_{1} \tilde{g}^{\alpha \beta} \tilde{g}^{\mu \nu}+c_{2} \tilde{g}^{\alpha \mu} \tilde{g}^{\beta \nu} \\
& +c_{3} \tilde{g}^{\alpha \nu} \tilde{g}^{\beta \mu}+c_{4} \tilde{g}^{\mu \nu} u^{\alpha} u^{\beta} .
\end{aligned}
$$

Equivalently, the Action Integral (6) can expressed in terms of the kinematic quantities $\left\{\tilde{\theta}, \tilde{\sigma}_{\mu \nu}, \tilde{\omega}_{\mu \nu}, \tilde{\alpha}^{\mu}\right\}$ as follows

$$
\begin{aligned}
& S_{A E}\left(g_{\mu \nu}, \tilde{\Gamma}_{\mu \nu}^{\kappa} ; u^{\mu}\right) \\
& \quad=\int \sqrt{-\tilde{g}} d x^{4}\left(\frac{c_{\theta}}{3} \tilde{\theta}^{2}+c_{\sigma} \tilde{\sigma}^{2}+c_{\omega} \tilde{\omega}^{2}+c_{\alpha} \tilde{\alpha}^{2}\right),
\end{aligned}
$$


where the new parameters $c_{\theta}, c_{\sigma}, c_{\omega}, c_{a}$ are functions of $c_{1}, c_{2}, c_{3}$ and $c_{4}$, that is, $c_{\theta}=\left(c_{1}+3 c_{2}+c_{3}\right), c_{\sigma}=$ $c_{1}+c_{3}, c_{\omega}=c_{1}-c_{3}, c_{a}=c_{4}-c_{1}$. The kinematic quantities giving the expansion rate $\tilde{\theta}$, the shear $\tilde{\sigma}^{2}=\tilde{\sigma}_{\mu \nu} \tilde{\sigma}^{\mu \nu}$, the vorticity $\tilde{\omega}^{2}=\tilde{\omega}_{\mu \nu} \tilde{\omega}^{\mu \nu}$ and the acceleration, $\tilde{\alpha}_{\mu}$ of the æther field are defined as

$$
\begin{aligned}
\tilde{\theta} & =\tilde{\nabla}_{\nu} u_{\mu} h^{\mu \nu}, \tilde{\sigma}_{\mu \nu}=\tilde{\nabla}_{(\lambda} u_{\kappa)}\left(h_{\mu}^{\kappa} h_{\nu}^{\lambda}-\frac{1}{3} \tilde{\theta} h_{\mu \nu}\right), \\
\tilde{\omega}_{\mu \nu} & =\tilde{\nabla}_{[\lambda} u_{\kappa]} h_{\mu}^{\kappa} h_{\nu}^{\lambda} \text { and } \tilde{\alpha}_{\mu}=u^{\nu} \tilde{\nabla}_{\nu} u_{\mu},
\end{aligned}
$$

in which $h_{\mu v}=g_{\mu v}-\left(u^{\kappa} u_{\kappa}\right) u_{\mu} u_{v}$ is the projective tensor for the field $u^{\mu}$.

From (3) we find the gravitational field equations to be

$$
\begin{aligned}
\tilde{G}_{\mu \nu} & +\tilde{\nabla}_{\nu}\left(\tilde{\nabla}_{\mu} \phi\right)-(2 \xi-1)\left(\tilde{\nabla}_{\mu} \phi\right)\left(\tilde{\nabla}_{\nu} \phi\right) \\
& +\xi g_{\mu \nu} g^{\kappa \lambda}\left(\tilde{\nabla}_{\kappa} \phi\right)\left(\tilde{\nabla}_{\lambda} \phi\right)-g_{\mu \nu} U(\phi)=T_{\mu \nu}^{a e},
\end{aligned}
$$

where $T_{a b}^{a e}$ is the energy momentum tensor which corresponds to the aether field and $\tilde{G}_{\mu \nu}$ is the Einstein tensor in Weyl theory, that is, $\tilde{G}_{\mu \nu}=\tilde{R}_{\mu \nu}-\frac{1}{2} \tilde{R} g_{\mu \nu}$. The right-hand side of Eq. (10) corresponds to the energy-momentum tensor of the æther field:

$$
\begin{aligned}
T_{\mu \nu}^{a e}= & 2 c_{1}\left(\tilde{\nabla}_{\mu} u^{\alpha} \tilde{\nabla}_{\nu} u_{\alpha}-\tilde{\nabla}_{\alpha} u_{\mu} \tilde{\nabla}_{\beta} u_{\nu} \tilde{g}^{\alpha \beta}\right) \\
& +2 \lambda u_{\mu} u_{v}+\tilde{K}_{\alpha \beta}^{\mu \beta} \tilde{\nabla}_{\mu} u^{\alpha} \tilde{\nabla}_{\beta} u^{\mu} \tilde{g}_{\mu \nu} \\
& -2\left[\tilde{\nabla}_{\alpha}\left(u_{(\mu} J^{\alpha}{ }_{\nu)}\right)+\tilde{\nabla}_{\alpha}\left(u^{\alpha} J_{(a v)}\right)-\tilde{\nabla}_{\alpha}\left(u_{(\mu} J_{v)}{ }^{\alpha}\right)\right] \\
& -2 c_{4}\left(\tilde{\nabla}_{\alpha} u_{\mu} u^{\alpha}\right)\left(\tilde{\nabla}_{\beta} u_{\nu} u^{\beta}\right)
\end{aligned}
$$

where $J^{\mu}{ }_{\nu}=-\tilde{K}^{\mu \beta}{ }_{\nu \alpha} \tilde{\nabla}_{\beta} u^{\alpha}$.

\subsection{FLRW spacetime}

In the case of a spatially flat FLRW geometry, with line element

$d s^{2}=-d t^{2}+a^{2}(t)\left(d x^{2}+d y^{2}+d z^{2}\right)$,

for the comoving aether field $u^{\mu}=\delta_{t}^{\mu}$ and for the line element (12) we calculate

$\tilde{\theta}=\theta-\dot{\phi}, \quad \tilde{\sigma}^{2}=0, \quad \tilde{\omega}^{2}=0$ and $\tilde{\alpha}^{2}=0$,

where $\theta$ is the Riemannian expansion rate defined as $\theta=3 \frac{\dot{a}}{a}$. The gravitational field equations are expressed as follows.

The 00 component of the field equations is

$\frac{\theta^{2}}{3}-\rho_{\phi}-\rho^{\mathfrak{x}}=0$,

while the rest of the nonzero components of the field equations are expressed as

$\dot{\theta}+\frac{\theta^{2}}{3}+\frac{1}{2}\left(\rho_{\phi}+3 p_{\phi}\right)+\frac{1}{2}\left(\rho^{\mathfrak{x}}+3 p^{\mathfrak{x}}\right)=0$, where $\rho_{\phi}, p_{\phi}$ are the energy density and pressure of the scalar field, that is,

$\rho_{\phi}(\phi, \dot{\phi})=\frac{\zeta}{2} \dot{\phi}^{2}-U(\phi), \quad p_{\phi}(\phi, \dot{\phi})=\frac{\zeta}{2} \dot{\phi}^{2}+U(\phi)$

where parameter $\zeta:=2 \xi-\frac{3}{2}$ is a coupling parameter between the scalar field and the gravity. Furthermore, $\rho^{\mathfrak{x}}, p^{\mathfrak{x}}$ are the density and pressure of the æther field, defined as

$\rho^{\mathfrak{x}}=-\frac{c_{\theta}}{3} \tilde{\theta}^{2}, p^{\mathfrak{x}}=\frac{c_{\theta}}{3}\left(2 \tilde{\theta}_{, t}+\tilde{\theta}^{2}\right)$,

that is,

$\rho^{\mathfrak{x}}=-\frac{c_{\theta}}{3}(\theta-\dot{\phi})^{2}, \quad p^{\mathfrak{x}}=\frac{c_{\theta}}{3}\left(2(\dot{\theta}-\ddot{\phi})+(\theta-\dot{\phi})^{2}\right)$.

By replacing in (14), (15) we derive the gravitational field equations

$$
\begin{aligned}
& \left(1+c_{\theta}\right) \frac{\theta^{2}}{3}-\frac{2}{3} c_{\theta} \theta \dot{\phi}-\left(\frac{\zeta}{2}-\frac{c_{\theta}}{3}\right) \dot{\phi}^{2}-U(\phi)=0 \\
& \left(1+c_{\theta}\right) \dot{\theta}+\frac{\left(1+c_{\theta}\right)}{3} \theta^{2}-\frac{2}{3} c_{\theta} \theta \dot{\phi} \\
& +\left(\zeta+\frac{c_{\theta}}{3}\right) \dot{\phi}^{2}-c_{\theta} \ddot{\phi}-U(\phi)=0,
\end{aligned}
$$

while the equation of motion for the scalar field is

$$
\begin{aligned}
& \left(2 c_{\theta}-3\left(1+c_{\theta}\right) \zeta\right) \ddot{\phi}+3 \zeta c_{\theta} \dot{\phi}^{2} \\
& \quad-3\left(1+c_{\theta}\right) \zeta \theta \dot{\phi}-3\left(1+c_{\theta}\right) U_{, \phi}=0 .
\end{aligned}
$$

In the following we investigate the existence of analytic solutions for the dynamical system (19)-(21). It is important to mention that the three equations are not independent, indeed equation (19) is a conservation law for the higherorder Eqs. (20), (21).

It is important to mention that in the latter dynamical system for $\zeta=0$ the system is degenerated and it has only one dependent variable, hence, in the following we consider that $\zeta \neq 0$.

\section{Exact solutions}

Before proceeding with the derivation of the exact solution we perform the following change of variable $\phi(t)=$ $-\frac{2}{3} \frac{c_{\theta}}{\zeta} \ln \psi(t)$, and define $V(\psi)=U\left(-\frac{2}{3} \frac{c_{\theta}}{\zeta} \ln \psi(t)\right)$, where now the gravitational field equations become

$$
\begin{aligned}
& \left(1+c_{\theta}\right) \frac{\theta^{2}}{3}+\frac{4}{9} \frac{\left(c_{\theta}\right)^{2}}{\zeta} \frac{\dot{\psi}}{\psi} \theta \\
& +\frac{2\left(c_{\theta}\right)^{2}\left(2 c_{\theta}-3 \zeta\right)}{27 \zeta^{2}}\left(\frac{\dot{\psi}}{\psi}\right)^{2}-V(\psi)=0, \\
& \left(1+c_{\theta}\right)\left(\dot{\theta}+\frac{1}{3} \theta^{2}\right)+\frac{4}{9} \frac{\left(c_{\theta}\right)^{2}}{\zeta} \frac{\dot{\psi}}{\psi} \theta
\end{aligned}
$$




$$
\begin{aligned}
& +\frac{2\left(c_{\theta}\right)^{2}\left(2 c_{\theta}-3 \zeta\right)}{27 \zeta^{2}}\left(\frac{\dot{\psi}}{\psi}\right)^{2}+\frac{2}{3} \frac{\left(c_{\theta}\right)^{2}}{\zeta} \frac{\ddot{\psi}}{\psi}-V(\psi)=0 \\
& \frac{4 c_{\theta}^{2}\left(3\left(c_{\theta}+1\right) \zeta-2 c_{\theta}\right)}{27\left(c_{\theta}+1\right) \zeta^{2}} \frac{\ddot{\psi}}{\psi}+\frac{4 c_{\theta}^{2}\left(2 c_{\theta}-3 \zeta\right)}{27 \zeta^{2}}\left(\frac{\dot{\psi}}{\psi}\right)^{2} \\
& +\frac{4 c_{\theta}^{2}}{9 \zeta} \frac{\dot{\psi}}{\psi} \theta+\psi V^{\prime}(\psi)=0
\end{aligned}
$$

We remark that in order for the field Eqs. (22)-(24) to be equivalent with those of (19)-(21) for a real field $\phi(t)$, we remark that by the definition of the coordinate transformation $\psi(t)=\exp \left(-\frac{3 \zeta}{2 c_{\theta}} \phi(t)\right)$ it follows that $\psi(t) \geq 0$. Point transformations does not affect the existence of solutions and the dynamical behaviour.

Finally, after some algebra, the gravitational equations (22), (23), (24) are reduced to

$$
\begin{aligned}
V(\psi)= & \frac{2 c_{\theta}^{2}\left(2 c_{\theta}-3 \zeta\right) \dot{\psi}^{2}}{27 \zeta^{2} \psi^{2}}+\frac{4 c_{\theta}^{2} \theta \dot{\psi}}{9 \zeta \psi}+\frac{1}{3}\left(c_{\theta}+1\right) \theta^{2}, \\
V^{\prime}(\psi)= & \frac{4 c_{\theta}^{2}\left(3 \zeta-2 c_{\theta}\right) \dot{\psi}^{2}}{27 \zeta^{2} \psi^{3}}-\frac{4 c_{\theta}^{2} \theta \dot{\psi}}{9 \zeta \psi^{2}} \\
& +\frac{2\left(3\left(c_{\theta}+1\right) \zeta-2 c_{\theta}\right) \dot{\theta}}{9 \zeta \psi}, \\
\ddot{\psi}= & -\frac{3\left(c_{\theta}+1\right) \zeta \psi \dot{\theta}}{2 c_{\theta}^{2}} .
\end{aligned}
$$

We proceed by study the existence of exact solution for the scalar field for specific forms of the scalar factor $a(t)$ which describes exact solutions of special interests.

\subsection{Power-law solution}

Consider the power-law solution $\theta(t)=\frac{2 c_{\theta}^{2}}{3\left(1+c_{\theta}\right) \zeta} \frac{\theta_{0}}{t}$, which describes a universe dominated by an ideal gas with constant equation of state parameter $w$ and scale factor $a(t)=$ $a_{0} t^{\frac{2}{3(1+w)}}$, with $w=-1+\frac{3 \zeta\left(1+c_{\theta}\right)}{\theta_{0} c_{\theta}^{2}}$, the solution describes an inflationary universe when $w<-\frac{1}{3}$, while in the special cases where $\theta_{0}=\frac{3 \zeta\left(1+c_{\theta}\right)}{c_{\theta}^{2}}, \theta_{0}=\frac{9 \zeta\left(1+c_{\theta}\right)}{4 c_{\theta}^{2}}$ or $\theta_{0}=\frac{3 \zeta\left(1+c_{\theta}\right)}{2 c_{\theta}^{2}}$, the ideal gas is that of dust fluid, radiation or stiff fluid respectively.

By replacing in (22) and (23) we calculate for the scalar field

$$
\begin{aligned}
& \psi(t)=\psi_{1} t^{p_{+}}+\psi_{2} t^{p_{-}}, \quad p_{ \pm}=\frac{1}{2}\left(1 \pm \sqrt{1+4 \theta_{0}}\right) \\
& \frac{27\left(1+c_{\theta}\right) \zeta^{2}}{2 c_{\theta}^{2}} t^{2} \psi V(\psi(t)) \\
& =2\left(c_{\theta}\right)^{2}\left(\psi_{1}\left(p_{+}+\theta_{0}\right) t^{p_{+}}+\psi_{2}\left(p_{-}+\theta_{0}\right) t^{p_{-}}\right) \\
& \quad+\left(2 c_{\theta}-3\left(1+c_{\theta}\right) \zeta\right)\left(p_{1} \psi_{1} t^{p_{+}}+p_{2} \psi_{2} t^{p_{-}}\right)^{2} .
\end{aligned}
$$

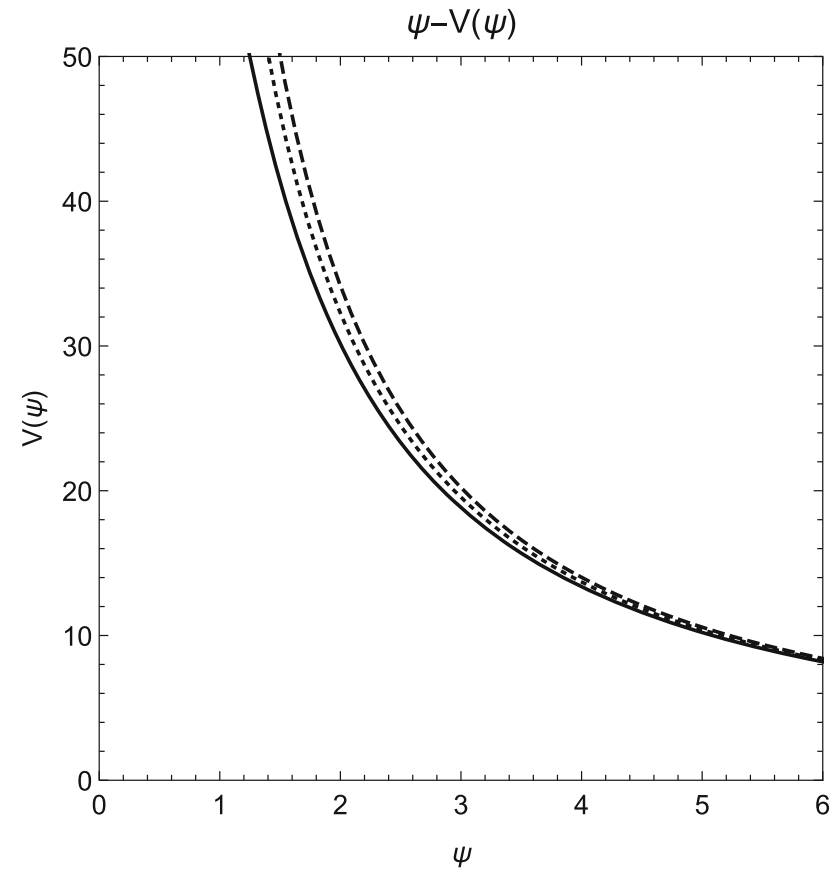

Fig. 1 Qualitative behaviour of the scalar field potential $V(\psi)$ for various values of the free parameters $\psi_{1}, \psi_{2}$. Solid line is for $\left(\psi_{1}, \psi_{2}\right)=\left(\frac{1}{2}, \frac{1}{2}\right)$, dotted line is for $\left(\psi_{1}, \psi_{2}\right)=\left(\frac{1}{2}, 0\right)$, dashed line is for $\left(\psi_{1}, \psi_{2}\right)=\left(\frac{1}{2},-\frac{1}{2}\right)$. From the plots we observe that the potential behaves like a power-law function. The plots are for $\theta_{0}=1, \zeta=1$ and $c_{\theta}=6$ where the power-law solution describes an accelerated universe. The potentials are for the power-law scale factor

In Fig. 1, we present the parametric plot for the scalar field potential $V(\psi)$ as it is expressed by (29). In the special limiting case where $\psi_{1} \psi_{2}=0$, lets say that $\psi_{2}=0$, the exact solution for the scalar field potential becomes $V(\psi)=\frac{V_{0}^{A}}{t^{2}}$ with $V_{0}^{A}=\frac{2 c_{\theta}^{2} \zeta^{2}\left(c_{\theta} p_{+}(2-3 \zeta)-3 p_{+}^{2} \zeta+2 c_{\theta}^{2}\left(p_{1}+\theta_{0}\right)\right)}{27\left(1+c_{\theta}\right)}$; thus, we end up with the power-law potential

$V(\psi)=V_{0}^{A}\left(\psi_{1}\right)^{\frac{2}{p_{+}}} \psi^{-\frac{2}{p_{+}}}$.

\section{2 de Sitter spacetime}

We assume now that the expansion rate $\theta(t)$ is a constant, i.e. $\theta(t)=\theta_{0}$. That solution describes the de Sitter universe with scale factor $a(t)=a_{0} e^{\frac{\theta_{0}}{3} t}$. Hence from (22) and (23) we find the closed-form solution for the scalar field

$\psi(t)=\psi_{1}\left(t-t_{0}\right)$

where $\psi_{1}, t_{0}$ are integration constants; for the scalar field potential it follows

$$
\begin{aligned}
V(\psi(t))= & \frac{\left(1+c_{\theta}\right)}{3} \theta_{0}^{2}+\frac{4 c_{\theta}^{2}}{9 \zeta} \frac{\theta_{0}}{\left(t-t_{0}\right)} \\
& +\frac{2\left(c_{\theta}\right)^{2}\left(2 c_{\theta}-3 \zeta\right)}{27 \zeta^{2}\left(t-t_{0}\right)^{2}} .
\end{aligned}
$$


Finally we end up with the functional form of the potential

$V(\psi(t))=V_{0}^{B}+V_{1}^{B} \psi^{-1}+V_{1}^{C} \psi^{-2}$.

where $V_{0}^{B}=\frac{\left(1+c_{\theta}\right)}{3} \theta_{0}^{2}, \quad V_{1}^{B}=\frac{4 c_{\theta}^{2}}{9 \zeta} \psi_{1} \theta_{0}$ and $V_{1}^{C}=$ $\frac{2\left(c_{\theta}\right)^{2}\left(2 c_{\theta}-3 \zeta\right)}{27 \zeta^{2}}\left(\psi_{1}\right)^{2}$.

\subsection{Quadratic Lagrangian inflation}

Let us consider the scale factor $a(t)=a_{0} \exp \left(-a_{1} t^{2}\right)$ which describes an exact solution of Einstein's General Relativity with quadratic corrections terms [63]. This solution can also be recovered by a modified Chaplygin gas in General Relativity [64]. For this scale factor we calculate $\theta(t)=-\frac{2 \theta_{0}}{3\left(1+c_{\theta}\right) \zeta} t$, where we have set $a_{1}=-\frac{\theta_{0} c_{\theta}^{2}}{9\left(1+c_{\theta}\right) \zeta}$.

Therefore, from the field Eqs. (22) and (23) it follows

$$
\begin{aligned}
& \psi(t)=\psi_{1} e^{\sqrt{\theta_{0}} t}+\psi_{2} e^{-\sqrt{\theta_{0}} t}, \\
& \frac{27\left(1+c_{\theta}\right) \zeta^{2}}{2 \theta_{0} c_{\theta}^{2}}\left(e^{2 \sqrt{\theta_{0}} t} \psi_{1}+\psi_{2}\right)^{2} V(\psi(t)) \\
& =2 c_{\theta}^{2}\left(e^{2 \sqrt{\theta_{0}} t}\left(\sqrt{\theta_{0}} t-1\right) \psi_{1}+\psi_{2}\left(\sqrt{\theta_{0} t}+1\right)\right)^{2} \\
& \quad+\left(3\left(1+c_{\theta}\right) \zeta-2 c_{\theta}\right)\left(e^{2 \sqrt{\theta_{0}} t} \psi_{1}-\psi_{2}\right)^{2} .
\end{aligned}
$$

For $\psi_{2}=0$, the scalar field potential is written as

$V(\psi)=V_{0}^{C}+V_{1}^{C} \ln \left(\frac{\psi}{\psi_{1}}\right)+V_{2}^{C}\left(\ln \left(\frac{\psi}{\psi_{1}}\right)\right)^{2}$,

where $V_{0}^{C}=\frac{2 \theta_{0}\left(2 c_{\theta}\left(1+c_{\theta}\right)-3 \zeta\right)}{27\left(1+c_{\theta}\right) \zeta^{2}}, V_{1}^{C}=-\frac{8 \theta_{0} c_{\theta}^{4}}{27\left(1+c_{\theta}\right) \zeta^{2}}$ and $V_{2}^{C}=\frac{4 \theta_{0} c_{\theta}^{4}}{27\left(1+c_{\theta}\right) \zeta^{2}}$.

\subsection{Scale factor $a(t)=a_{0} t^{\alpha_{1}} e^{\alpha_{2} t}$}

Scale factor of the form $a(t)=a_{0} t^{\alpha_{1}} e^{\alpha_{2} t}$ has been studied before in [64]. For this solution we find $\theta(t)=3\left(\frac{a_{1}}{t}+a_{2}\right)$, and $\dot{\theta}(t)=3 \frac{a_{1}}{t}$. For simplicity we replace $a_{1}=\frac{\theta_{0} c_{\theta}^{2}}{9 \zeta\left(1+c_{\theta}\right)}$, while for the scalar field it follows

$$
\begin{aligned}
& \psi(t)=\psi_{1} t^{q_{+}}+\psi_{2} t^{q_{-}}, q_{ \pm}=\frac{1}{2}\left(1+\sqrt{1+2 \theta_{0}}\right), \\
& 27 t^{2} \zeta^{2} V(\psi(t))=4 c_{\theta}^{3} q_{-}^{2}-6 c_{\theta}^{2} q_{-}\left(q_{-}-6 a_{2} t\right) \zeta \\
& +81 a_{2}\left(1+c_{\theta}\right) t^{2} \zeta^{2}+\frac{2 c_{\theta}^{2}\left(2 c_{\theta}^{2} q_{-}+9 a_{2}\left(1+c_{\theta}\right) t \zeta\right)}{1+c_{\theta}} \theta_{0} \\
& +\frac{c_{\theta}^{4}}{1+c_{\theta}}+ \\
& +\frac{4 c_{\theta}^{2}\left(q_{+}-q_{-}\right) t^{q_{+}}\left(\left(1+c_{\theta}\right)\left(2 c_{\theta} q_{-}-3 q_{-} \zeta+9 a_{2} t \zeta\right)+\theta_{0} c_{\theta}^{2}\right)}{\left(1+c_{\theta}\right) \psi(t)} \psi_{1} \\
& +\frac{2 c_{\theta}^{2}\left(q_{+}-q_{-}\right) t^{2 q_{1}}\left(2 c_{\theta}-3 \zeta\right)}{\psi(t)^{2}} \psi_{1}^{2} .
\end{aligned}
$$

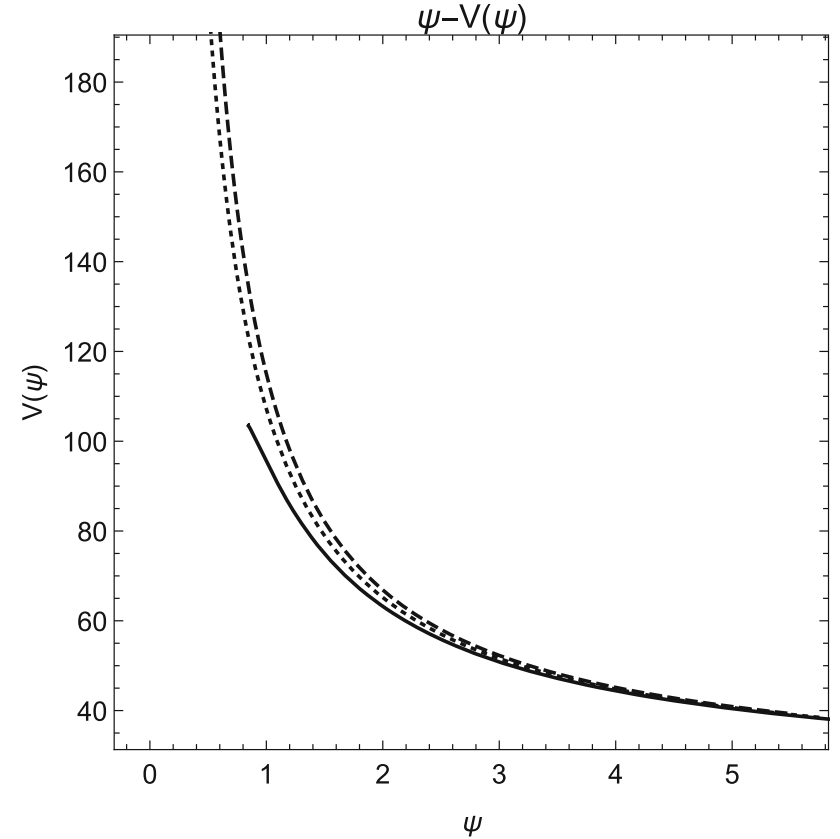

Fig. 2 Qualitative behaviour of the scalar field potential $V(\psi)$ for various values of the free parameters $\psi_{1}, \psi_{2}$. Solid line is for $\left(\psi_{1}, \psi_{2}\right)=\left(\frac{1}{2}, \frac{1}{2}\right)$, dotted line is for $\left(\psi_{1}, \psi_{2}\right)=\left(\frac{1}{2}, 0\right)$, dashed line is for $\left(\psi_{1}, \psi_{2}\right)=\left(\frac{1}{2},-\frac{1}{2}\right)$. From the plots we observe that the potential behaves like a power-law function. The plots are for $\theta_{0}=1, \zeta=1$ and $c_{\theta}=6$ and $a_{2}=1$. The potential is for the scale factor $a(t)=a_{0} t^{\alpha_{1}} e^{\alpha_{2} t}$

For $\psi_{2}=0$, it follows

$V(\phi)=V_{0}^{D}+V_{1}^{D}\left(\frac{\psi}{\psi_{1}}\right)^{-\frac{1}{q_{+}}}+V_{2}^{D}\left(\frac{\psi}{\psi_{2}}\right)^{-\frac{2}{q_{+}}}$,

where $V_{0}^{D}=3 a_{2}^{2}\left(1+c_{\theta}\right), V_{1}^{D}=\frac{2 a_{2} c_{\theta}^{2}\left(2 q_{+}+\theta_{0}\right)}{3 \zeta}$ and $V_{2}^{D}=$ $\frac{2 c_{\theta}^{2}\left(1+c_{\theta}\right) q_{+}^{2}\left(2 c_{\theta}-3 \zeta\right)+4 c_{\theta}^{4} q_{+} \theta_{0}+c_{\theta}^{4} \theta_{0}^{2}}{27\left(1+c_{\theta}\right) \zeta^{2}}$. The parametric plot of the scalar field potential $V(\psi)$ is presented in Fig. 2 for various values of the free parameters.

\subsection{Intermediate inflation}

Consider the power-law solution $\theta(t)=\frac{1}{3} A f t^{-(1-f)}, A>$ $0,0<f<1$ which describes intermediate inflation with deceleration parameter $q=-1+\frac{(1-f)}{A f} t^{-f}$ and scale factor $a(t)=a_{0} e^{A t^{f}}$. The solution describes an inflationary universe [40-42]. The expansion of the universe with this scale factor is slower than the de Sitter inflation $\left(a(t)=a_{0} e^{\frac{\theta_{0}}{3} t}\right)$, but faster than the power law inflation $\left(a(t)=a_{0} t^{q}\right.$ where $q>1$ ). It was shown that the intermediate inflation arises as the slow-roll solution to potentials which fall off asymptotically as an inverse power law inflation in the standard canonical framework and can be modelled by an exact cosmological solution [41,42]. The intermediate inflation has also been studied in some warm inflationary scenarios in 
order to examine its predictions for inflationary observables [43-46]. With these assumptions the field equations (22), (23), (24) becomes:

$$
\begin{aligned}
V(\psi(t))= & \frac{1}{27} A^{2}\left(c_{\theta}+1\right) f^{2} t^{2 f-2}+\frac{4 A c_{\theta}^{2} f t^{f-1} \dot{\psi}}{27 \zeta \psi(t)} \\
& +\frac{2 c_{\theta}^{2}\left(2 c_{\theta}-3 \zeta\right) \dot{\psi}^{2}}{27 \zeta^{2} \psi^{2}}, \\
V^{\prime}(\psi)= & -\frac{4 A c_{\theta}^{2} f t^{f-1} \dot{\psi}}{27 \zeta \psi^{2}} \\
& +\frac{2 A(f-1) f\left(3\left(c_{\theta}+1\right) \zeta-2 c_{\theta}\right) t^{f-2}}{27 \zeta \psi} \\
& +\frac{4 c_{\theta}^{2}\left(3 \zeta-2 c_{\theta}\right) \dot{\psi}^{2}}{27 \zeta^{2} \psi^{3}}, \\
\ddot{\psi}= & -\frac{A\left(c_{\theta}+1\right)(f-1) f \zeta t^{f-2} \psi}{2 c_{\theta}^{2}} .
\end{aligned}
$$

Choosing $0<f<1, A>0$, we obtain the exact solution

$$
\begin{aligned}
\psi= & 2^{-\frac{1}{2 f}} \sqrt{t}\left(c_{\theta} f\right)^{-1 / f}\left(-A^{2}(f-1) f \zeta\right)^{\frac{1}{2 f}}\left|c_{\theta}+1\right|^{\frac{1}{2 f}} \\
& \times\left[\psi_{1} \Gamma\left(\frac{f-1}{f}\right)\right. \\
& \times I_{-\frac{1}{f}}\left(\frac{\sqrt{2} A t^{f / 2} \sqrt{-(f-1) f \zeta} \sqrt{\left|c_{\theta}+1\right|}}{c_{\theta} f}\right) \\
& +\psi_{2}(-1)^{\frac{1}{f}} \Gamma\left(1+\frac{1}{f}\right) \\
& \left.\times I_{\frac{1}{f}}\left(\frac{\sqrt{2} A t^{f / 2} \sqrt{-(f-1) f \zeta} \sqrt{\left|c_{\theta}+1\right|}}{c_{\theta} f}\right)\right]
\end{aligned}
$$

where $\psi_{1}, \psi_{2}$ are integration constants and $I_{-1 / f}$ and $I_{1 / f}$ denote Bessel functions. Considering the condition $0<f<$ 1 we set $\psi_{1}=0$ to obtain real solutions. In Fig. 3 the qualitative behaviour of the latter scalar field potential is presented.

\subsection{Log-mediate inflation}

Consider the solution $\theta(t)=\frac{A \lambda \ln ^{\lambda-1}(t)}{3 t}$, where $\lambda$ and $A$ are dimensionless constant parameters such that $\lambda>1$ and $A>0$, with deceleration parameter $q=-1+\frac{\ln ^{1-\lambda}(t)}{A \lambda}-$ $\frac{(\lambda-1) \ln ^{-\lambda}(t)}{A \lambda}$ and scale factor $a(t)=\exp \left[A(\ln t)^{\lambda}\right]$. This generalized model of the expansion of the universe is called $\log$-mediate inflation $[42,46]$. Note that for the special case in which $\lambda=1, A=p$, the log-mediate inflation model becomes a power-law inflation model [47].

With these assumptions the field Eqs. (22), (23), (24) becomes:

$$
V(\psi(t))=\frac{A^{2}\left(c_{\theta}+1\right) \lambda^{2} \ln ^{2 \lambda-2}(t)}{27 t^{2}}
$$

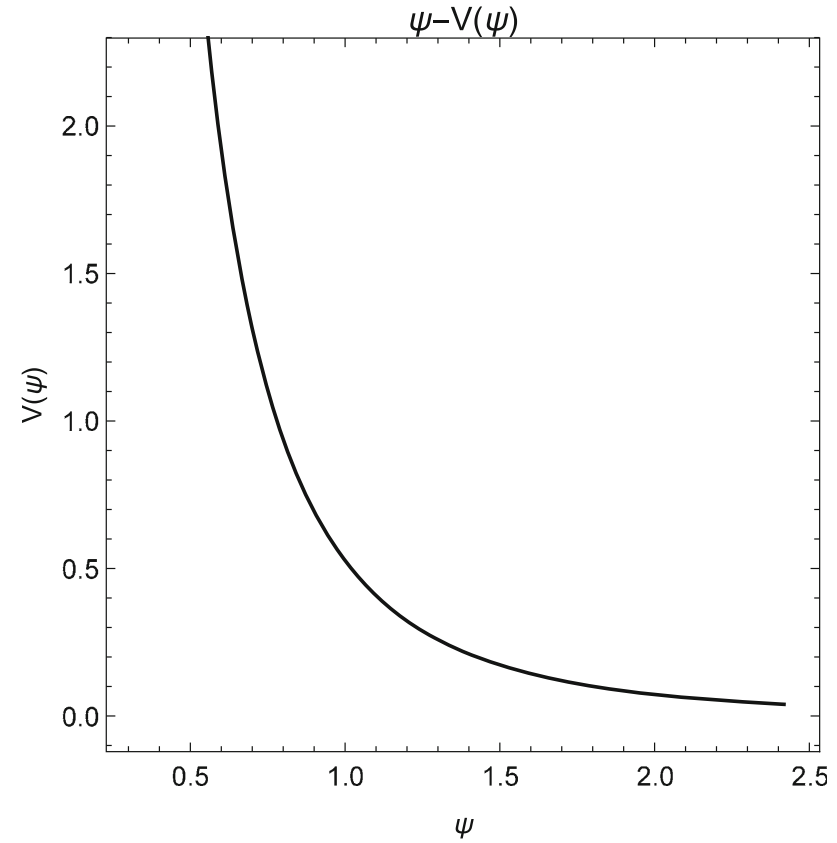

Fig. 3 Qualitative behaviour of the scalar field potential $V(\psi)$ for the intermediated inflation model, plot is for $\left(\psi_{1}, \psi_{2}, c_{\theta}, \zeta, f, A\right)=$ $(0,2,1,-1,1 / 2,1)$

$$
\begin{aligned}
& +\frac{4 A c_{\theta}^{2} \lambda \ln ^{\lambda-1}(t) \dot{\psi}}{27 \zeta t \psi} \\
& +\frac{2 c_{\theta}^{2}\left(2 c_{\theta}-3 \zeta\right) \dot{\psi}^{2}}{27 \zeta^{2} \psi^{2}} \\
V^{\prime}(\psi(t))= & -\frac{4 A c_{\theta}^{2} \lambda \ln ^{\lambda-1}(t) \dot{\psi}}{27 \zeta t \psi^{2}} \\
& +\frac{2 A \lambda\left(3\left(c_{\theta}+1\right) \zeta-2 c_{\theta}\right)(\lambda-\ln (t)-1) \ln ^{\lambda-2}(t)}{27 \zeta t^{2} \psi} \\
& +\frac{4 c_{\theta}^{2}\left(3 \zeta-2 c_{\theta}\right) \dot{\psi}^{2}}{27 \zeta^{2} \psi^{3}}, \\
\ddot{\psi}= & \frac{A\left(c_{\theta}+1\right) \zeta \lambda \psi \ln ^{\lambda-2}(t)(-\lambda+\ln (t)+1)}{2 c_{\theta}^{2} t^{2}}
\end{aligned}
$$

However, Eq. (46) is not integrable in closed form. We propose the asymptotic expansion

$$
\begin{aligned}
& \psi(t) \sim c_{0} t^{\alpha} \ln (t)+\epsilon\left(\psi_{2} t+\psi_{1}\right) \\
& t^{\alpha-\frac{1}{2}}+O\left(\epsilon^{2}\right), \text { for } t \epsilon<B \text { for some } B>0, \text { and } 0<\epsilon \ll 1
\end{aligned}
$$

The Eq. (46) becomes

$$
\begin{aligned}
0= & R(t, \alpha ; \epsilon, \lambda, \zeta) \\
:= & \frac{c_{0} t^{\alpha-2}(A \zeta \lambda(\lambda-\ln (t)-1) \ln (t)+\ln (t)(2 \alpha+(\alpha-1) \alpha \ln (t)-1))}{\ln (t)} \\
& +\epsilon t^{\alpha-\frac{5}{2}}\left(A \zeta \lambda\left(\psi_{2} t+\psi_{1}\right)(\lambda-\ln (t)-1) \ln ^{\lambda-2}(t)\right. \\
& \left.+\frac{1}{4}(2 \alpha-1)\left((2 \alpha-3) \psi_{1}+(2 \alpha+1) \psi_{2} t\right)\right)+O\left(\epsilon^{2}\right)
\end{aligned}
$$


For $\alpha \in\left\{1, \frac{1}{2}, 0,-\frac{1}{2},-\frac{5}{2}, \frac{1}{2}(1-\sqrt{1+4 \lambda})\right\}$, it is verified $\lim _{t \rightarrow \infty} R(t, \alpha ; \epsilon, \lambda, \zeta)=0$.

Moreover, setting $\alpha=\frac{3}{2}-k, k>0$, it follows where $\mu=\frac{\sqrt{c_{\theta}^{2}+12 \zeta\left(1+c_{\theta}\right)}}{2 c_{\theta}}$, and $\psi_{1}, \psi_{2}$ are two integration constants. For simplicity we omit the presentation of the exact form of the scalar field potential $V(\psi)$. Thus for specific values of the free parameters we present the parametric evolution of $V(\psi)$ in Fig. 4.

$$
\begin{aligned}
& R(t, \alpha ; \epsilon, \lambda, \zeta):=\frac{c_{0} t^{-k-\frac{1}{2}}(4 A \zeta \lambda(\lambda-\ln (t)-1) \ln \lambda(t)+\ln (t)((4(k-2) k+3) \ln (t)-8 k+8))}{4 \ln (t)} \\
& \quad+\frac{\epsilon t^{-k-1}\left(A \zeta \lambda\left(\psi_{2} t+\psi_{1}\right)(\lambda-\ln (t)-1) \ln ^{\lambda}(t)+(k-1) \ln ^{2}(t)\left(\psi_{2}(k-2) t+\psi_{1} k\right)\right)}{\ln ^{2}(t)}+O\left(\epsilon^{2}\right), \\
& \quad \lim _{t \rightarrow \infty} R(t, \alpha ; \epsilon, \lambda, \zeta)=0 .
\end{aligned}
$$

Hence, the gravitational field equations in Einstein-ætherWeyl theory in a spatially flat FLRW background space described by the set of differential Eqs. (19), (20), (21) with scale factor $a(t)=\exp \left[A(\ln t)^{\lambda}\right]$ admits an asymptotic solution

$\psi(t) \sim c_{0} t^{\alpha} \ln (t)$,

\section{Integrability of the gravitational field equations}

In Sect. 3 we solved the gravitational field equations for different scale factors, which are of interests as cosmological solutions. The exact solutions of our analysis have the sufficient number of initial constants of integration, they are the constants $\psi_{1}, \psi_{2}$ and the non-essential constant of the time translation $t \rightarrow t+t_{1}$ which we have omitted. Hence, the solutions that we have found are the general analytic

$V(\psi(t)) \sim \frac{A^{2}\left(c_{\theta}+1\right) \zeta^{2} \lambda^{2} \ln ^{2 \lambda}(t)+4 A c_{\theta}^{2} \zeta \lambda(\alpha \ln (t)+1) \ln ^{\lambda}(t)+2\left(2 c_{\theta}-3 \zeta\right)\left(\alpha c_{\theta} \ln (t)+c_{\theta}\right)^{2}}{27 \zeta^{2} t^{2} \ln ^{2}(t)}$,

as $t \rightarrow \infty$, for any $\alpha<\frac{3}{2}$.

That, is

$$
\begin{aligned}
& V \sim O\left(\left(\frac{1}{t}\right)^{2}\right)\left(\ln ^{2 \lambda}(t)+\ln ^{\lambda}(t)+1\right), \\
& \psi \sim t^{\alpha}\left(-c_{0} \ln \left(\frac{1}{t}\right)+O\left(\left(\frac{1}{t}\right)^{2}\right)\right) .
\end{aligned}
$$

\section{7 $\Lambda \mathrm{CDM}$ universe}

As a final application we consider the scale factor which describes the $\Lambda$-cosmology, i.e. $a(t)=a_{0} \sinh ^{\frac{2}{3}}(\omega t)$. Thus, for this exact solution the scalar field is found to be expressed in terms of the hypergeometric function

$$
\begin{aligned}
& \psi(t)=\psi_{1}(\tanh (\omega t))^{\frac{1}{2}-\mu} \\
& \times{ }_{2} F_{1}\left(\frac{1}{4}-\frac{\mu}{2}, \frac{3}{4}-\frac{\mu}{2} ; 1-\mu ; \tanh ^{2}(\omega t)\right) \\
& +\psi_{2}(\tanh (\omega t))^{\frac{1}{2}+\mu} \\
& \times{ }_{2} F_{1}\left(\frac{1}{4}+\frac{\mu}{2}, \frac{3}{4}+\frac{\mu}{2} ; 1+\mu ; \tanh ^{2}(\omega t)\right),
\end{aligned}
$$

solutions of the nonlinear dynamical system which provide these specific scale factors. Note, that we have not considered any functional form for the scalar field potential but for all the cases that we have studied, a scalar field potential can be found. Our analysis is motivated by the original work on cosmological solutions in scalar field theory by Ellis and Madsen [65]. There, the solutions that have been found are exact solutions and particularly, they are special solutions and not the complete solution of the dynamical system. Some analytic solutions in scalar field cosmology can be found by using techniques of analytic mechanics such is the theory of invariant transformations [66-68]. However in this study we have not applied any symmetry in order to find the solutions, that indicates that except from the constraint equation another conservation law should always exists for any functional form of the scalar field potential.

The new scalar field $\psi(t)=\exp \left(-\frac{3 \zeta}{2 c_{\theta}} \phi(t)\right)$ that we defined it was not an ad hoc selection. Indeed, in these coordinates by replacing $V(\psi)$ from (22) in (23) we end up with the second-order differential equation of the form

$\ddot{\psi}+\omega(t) \psi=0$, 


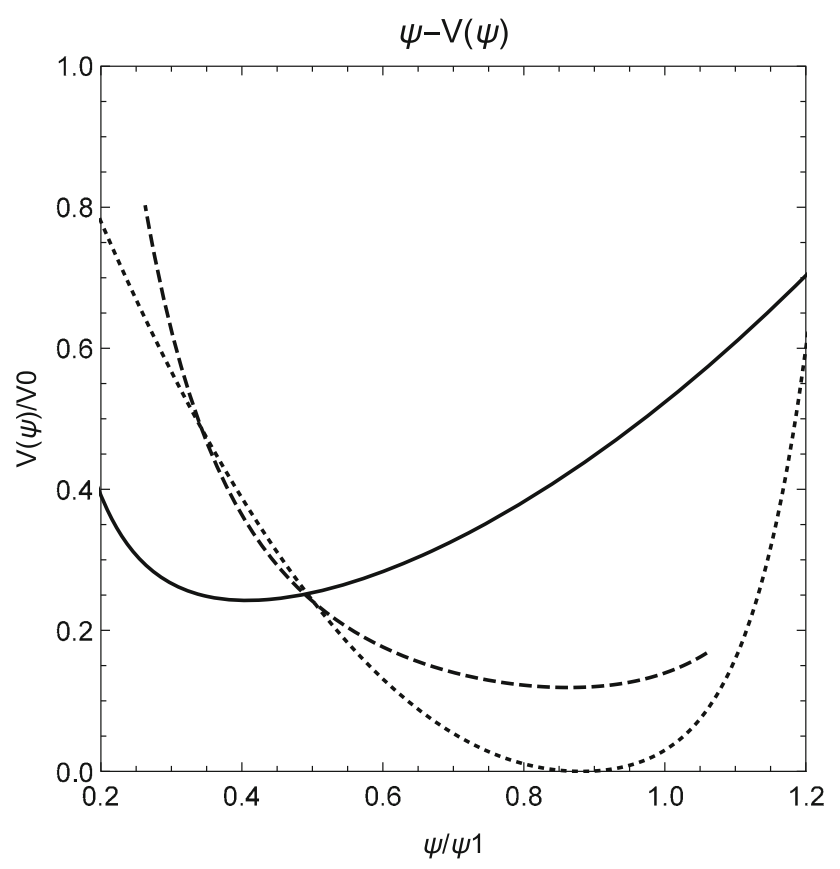

Fig. 4 Qualitative behaviour of the scalar field potential $V(\psi)$ for various values of the free parameters $\psi_{1}, \psi_{2}$. Solid line is for $\left(\psi_{1}, \psi_{2}\right)=\left(\frac{1}{2}, \frac{1}{2}\right)$, dotted line is for $\left(\psi_{1}, \psi_{2}\right)=\left(\frac{1}{2}, 0\right)$, dashed line is for $\left(\psi_{1}, \psi_{2}\right)=\left(\frac{1}{2},-\frac{1}{2}\right)$. From the plots we observe that the potential behaves like a power-law function. The plots are for $\omega=1, \zeta=1$ and $c_{\theta}=20$. The axes has been normalized. The potential is for the $\Lambda \mathrm{CDM}$ scale factor

where $\omega(t)=\left(\frac{3}{2} \frac{\left(1+c_{\theta}\right)}{c_{\theta}^{2}} \zeta \dot{\theta}\right)$.

The second-order differential equation is a linear equation also known as the time-dependent oscillator [69]. The differential equation (56) admits the conservation law [70]

$I=\frac{1}{2}\left((y \dot{\psi}-\dot{y} \psi)^{2}+\left(\frac{\psi}{y}\right)^{2}\right)$,

where $y=y(t)$ is any solution of the Ermakov-Pinney equation

$\ddot{y}+\omega(t) y-y^{-3}=0$.

Conservation law (57) it is known as Lewis invariant and it was derived for the first time as an adiabatic invariant [71]. Alternatively, the conservation law (57) can be constructed through a set of canonical transformations [72] or with the use of Noether's theorem [69]. The set of equations (56)-(58) it is also known as the Ermakov system which can be found in many applications in physical science [73-76].

Hence, for the gravitational field equations (19)-(21) the following theorem holds.

Theorem The gravitational field equations in Einsteinather-Weyl theory in a spatially flat FLRW background space described by the set of differential equations (19)-(20) form an integrable dynamical system for arbitrary potential. The two conservation laws are the constraint equation (19) and the Lewis invariant

$I(\phi, \dot{\phi}, y)=\frac{1}{2} e^{-\frac{3 \zeta}{c_{\theta}} \phi}\left(\left(\frac{3 \zeta}{2 c_{\theta}} y \dot{\phi}+\dot{y}\right)^{2}+y^{-2}\right)$,

where $y(t)$ satisfies the Ermakov-Pinney equation (58).

It is important to mention at this point that in another lapse function $d t=N(\tau) d \tau$ in the metric tensor (12) our results are valid. In such a case, the equivalent equation (56) is of the form

$\frac{d^{2} \psi}{d \tau^{2}}+\alpha(\tau) \frac{d \psi}{d \tau}+\beta(\tau) \psi=0$,

which also admits an invariant function [72] similar to the Lewis invariant.

Except from the Lewis invariant, the linear differential equation (60) is maximally symmetric and admits eight Lie point symmetries which form the $S L(3, R)$ Lie algebra [77], for arbitrary functions $\alpha(\tau)$ and $\beta(t)$. Hence, according to $\mathrm{S}$. Lie theorem the differential equation (60) is equivalent to the free particle $Y^{\prime \prime}=0$ and there exists a point transformation $\{\tau, \psi(\tau)\} \rightarrow\{\chi, Y(\chi)\}$ which transforms equation (60) into that of the free particle. For more details, we refer the reader to the review article [78]. That is an alternative way to prove the integrability of the gravitational field equations for the cosmological model of our consideration.

\section{Conclusions}

In this work we considered a spatially flat FLRW background space in Einstein-æther theory defined in Weyl integrable geometry. The novelty of this approach is that a scalar field coupled to the æther field is introduced in a geometric way. For this model we investigated the existence of exact solutions of special interests, in particular we focused on exact solutions which can describe the inflationary epoch of our universe.

Indeed, we proved that the cosmological model of our consideration can provide exact solutions such as the power-law inflation, de Sitter expansion, quadratic Lagrangian inflation and others. For these specific scale factors we were able to calculate the closed-form expression of the scalar field solution and of the scalar field potential.

Moreover, we investigate also the possibility of Einsteinæther-Weyl cosmological model to admit a cosmological solution where the scalar field unify the dark matter and the dark energy of the universe, and for that investigation we proved that there exists a scalar field potential which can describe explicitly the $\Lambda$ CDM universe. Scalar field models which unify the dark components of the universe have been drawn the attention of the academic society because they 
provide a simple mechanism for the observable universe, see [79-83] and references therein.

However, the main result of this work is that we were able to prove the integrability of the field equations of our cosmological model for arbitrary potential function. In particular we found a point transformation which reduce one of the two equations to the linear equation of the time-dependent oscillator, and to prove that the Lewis invariant is a conservation law for the field equations for arbitrary scalar field potential. This is an interesting result which we did not expect it, assuming the nonlinearity form of the field equations and mainly that according to our knowledge there is not any effective Lagrangian description for the cosmological field equations in order to apply techniques for the investigation of conservation laws similar with that applied before for the quintessence or the scalar tensor theories.

From this work it is clear that in the background space the Einstein-æther-Weyl cosmological model is cosmologically viable. Thus in a future work we plan to investigate further the physical properties of this theory as an inflationary model and as a unified model for the dark components of the universe.

Acknowledgements AP \& GL were funded by Agencia Nacional de Investigación y Desarrollo-ANID through the program FONDECYT Iniciación Grant No. 11180126. Additionally, GL is supported by Vicerrectoría de Investigación y Desarrollo Tecnológico at Universidad Católica del Norte.

Data Availability Statement This manuscript has no associated data or the data will not be deposited. [Authors' comment: This is a Theoretical Research Project.]

Open Access This article is licensed under a Creative Commons Attribution 4.0 International License, which permits use, sharing, adaptation, distribution and reproduction in any medium or format, as long as you give appropriate credit to the original author(s) and the source, provide a link to the Creative Commons licence, and indicate if changes were made. The images or other third party material in this article are included in the article's Creative Commons licence, unless indicated otherwise in a credit line to the material. If material is not included in the article's Creative Commons licence and your intended use is not permitted by statutory regulation or exceeds the permitted use, you will need to obtain permission directly from the copyright holder. To view a copy of this licence, visit http://creativecomm ons.org/licenses/by/4.0/.

Funded by SCOAP ${ }^{3}$.

\section{References}

1. T. Clifton, P.G. Ferreira, A. Padilla, C. Skordis, Phys. Rep. 513, 1 (2012)

2. Y. Fujii, K.-I. Maeda, The Scalar-Tensor Theory of Gravitation (Cambridge University Press, New York, 2003)

3. P. Horava, Phys. Rev. D 79, 084008 (2009)

4. W. Donnelly, T. Jacobson, Phys. Rev. D 82, 081501 (2010)

5. W. Donnelly, T. Jacobson, Phys. Rev. D 82, 064032 (2010)

6. S.M. Carroll, E.A. Lim, Phys. Rev. D 70, 123525 (2004)

7. R.G. Cai, L.M. Cao, N. Ohta, Phys. Lett. B 679, 504 (2009)
8. T. Christodoulakis, N. Dimakis, J. Geom. Phys. 62, 2401 (2012)

9. E.N. Saridakis, Eur. Phys. J. C 67, 229 (2010)

10. E. Kiritsis, G. Kofinas, Nucl. Phys. B 821, 467 (2009)

11. H. Lu, J. Mei, C.N. Pope, Phys. Rev. Lett. 103, 091301 (2009)

12. N.A. Nilsson, E. Czuchry, Phys. Dark Univ. 23, 100253 (2019)

13. I. Carruthers, T. Jacobson, Phys. Rev. D 83, 024034 (2011)

14. T.G. Zlosnik, P.G. Ferreira, G.D. Starkman, Phys. Rev. D 75, 044017 (2007)

15. C. Eling, T. Jacobson, M.Coleman Miller, Phys. Rev. D 76, 042003 (2007)

16. A.A. Coley, G. Leon, P. Sandin, J. Latta, JCAP 12, 010 (2015)

17. J. Latta, G. Leon, A. Paliathanasis, JCAP 11, 051 (2016)

18. A. Coley, G. Leon, Gen. Relativ. Gravit. 51, 115 (2019)

19. G. Leon, A. Coley, A. Paliathanasis, Ann. Phys. 412, 168002 (2020)

20. M. Roumeliotis, A. Paliathanasis, P.A. Terzis, T. Christodoulakis, EPJC 79, 349 (2019)

21. C. Ranjit, P. Rudraand, S. Kundu, EPJP 129, 208 (2014)

22. A.B. Balakin, J.P.S. Lemos, Ann. Phys. 350, 454 (2014)

23. H. Wei, X.-P. Yan, Y.-N. Zhou, Gen. Relativ. Gravit. 46, 1719 (2014)

24. B. Alhulaimi, R.J. van den Hoogen, A.A. Coley, JCAP 17, 045 (2017)

25. A. Wang, D. Wands, R. Maartens, JCAP 03, 013 (2010)

26. A.E. Gumrukcuoglu, S. Mukohyama, A. Wang, Phys. Rev. D 85, 064042 (2012)

27. G. Leon, A. Paliathanasis, EPJC 79, 746 (2019)

28. A.N. Tawfik, A.M. Diab, E.A. Dahab, IJMPA 31, 1650042 (2016)

29. S. Kanno, J. Soda, Phys. Rev. D 74, 063505 (2006)

30. T. Jacobson, Phys. Rev. D 89, 081501 (2014)

31. J.D. Barrow, Phys. Rev. D 85, 047503 (2012)

32. A.R. Solomon, J.D. Barrow, Phys. Rev. D 89, 024001 (2014)

33. A. Paliathanasis, G. Papagiannopoulos, S. Basilakos, J.D. Barrow, EPJC 79, 723 (2019)

34. G. Leon, A. Paliathanasis, N. Dimakis, EPJC 80, 1149 (2020)

35. R. Chan, M.F.A. da Silva, V.H. Satheeshkumar, Spherically symmetric analytic solutions and naked singularities in Einstein-Aether theory (2020). arXiv:2003.00227

36. M. Campista, R. Chan, M.F.A. da Silva, O. Goldoni, V.H. Satheeshkumar, J.F. Villas da Rocha, Can. J. Phys. 98, 917 (2020)

37. A. Paliathanasis, G. Leon, J.D. Barrow, EPJC 80(12), 1099 (2020)

38. C. Romero, J.B. Fonseca-Neto, M.L. Pucheu, Class. Quantum Gravity 29, 155015 (2012)

39. V. Perlick, Class. Quantum Gravity 8, 1369 (1991)

40. J.D. Barrow, Phys. Lett. B 235, 40 (1990)

41. J.D. Barrow, A.R. Liddle, Phys. Rev. D 47, 5219 (1993)

42. J.D. Barrow, N.J. Nunes, Phys. Rev. D 76, 043501 (2007)

43. S. del Campo, R. Herrera, JCAP 04, 005 (2009)

44. M. Jamil, D. Momeni, R. Myrzakulov, Int. J. Theor. Phys. 54, 1098 $1112(2015)$

45. R. Herrera, M. Olivares, N. Videla, IJMPD 23, 1450080 (2014)

46. R. Herrera, N. Videla, M. Olivares, Eur. Phys. J. C 78(11), 934 (2018)

47. F. Lucchin, S. Matarrese, Phys. Rev. D 32, 1316 (1985)

48. R. Aguila, J.E. MadrizAguilar, C. Moreno, M. Bellini, Eur. Phys. J. C 74, 3158 (2014)

49. M. Konstantinov, V. Melnikov, Int. J. Mod. Phys. D 4, 339 (1995)

50. J. Villanueva, F. Tapia, M. Molina, M. Olivares, Eur. Phys. J. C 78, 853 (2018)

51. J.E. Madriz Aguilar, C. Romero, Int. J. Mod. Phys. A 24, 1505 (2009)

52. J.E. Madriz Aguilar, C. Romero, J.B. Fonseca Neto, T.S. Almeida, J.B. Formiga, Class. Quantum Gravity 32, 215003 (2015)

53. K.A. Bonnikov, MYu. Konstantinov, V.N. Melnikov, Gravit. Cosmol. 1, 60 (1995)

54. J.M. Salim, S. Sautu, Class. Quantum Gravity 15, 203 (1998)

55. J.M. Salim, S. Sautu, Class. Quantum Gravity 16, 3281 (1999) 
56. T.S. Almeida, M.L. Puckeu, C. Romero, J.B. Formiga, Phys. Rev. D 89, 064047 (2014)

57. A. Paliathanasis, G. Leon, J.D. Barrow, EPJC 80, 731 (2020)

58. J.M. Salim, S.L. Sautu, Class. Quantum Gravity 13, 353 (1996)

59. A. Goriely, Integrability and Nonintegrability of Dynamical Systems, Advanced Series in Nonlinear Dynamics, vol. 19 (World Scientific Press, Singapore, 2001)

60. X. Zhang, Integrability of Dynamical Systems: Algebra and Analysis, Developments in Mathematics (Springer Nature Singapore Pte Ltd., Singapore, 2017)

61. F.M. Arscott, Periodic Differential Equations (Pergamon Press, Oxford, 1964)

62. E. Garcia-Rio, P. Gilkey, S. Nikcevic, R. Vazquez-Lorenzo, Applications of Affine and Weyl Geometry (Synthesis Lectures on Mathematics and Statistics) (Morgan and Claypool, San Rafael, 2013)

63. T.V. Ruzmaikina, A.A. Ruzmaikin, Sov. Phys. JETP 30, 372 (1970)

64. J.D. Barrow, A. Paliathanasis, Phys. Rev. D 94, 083518 (2016)

65. G.F.R. Ellis, M.S. Madsen, Class. Quantum Gravity 8, 667 (1991)

66. S. Basilakos, M. Tsampalis, A. Paliathanasis, Phys. Rev. D 83, $103512(2011)$

67. M. Tsamparlis, A. Paliathanasis, Symmetry 10, 233 (2018)
68. N. Dimakis, A. Karagiorgos, A. Zampeli, A. Paliathanasis, T. Christodoulakis, P.A. Terzis, Phys. Rev. D 93, 123518 (2016)

69. M. Lutzky, Phys. Lett. A 68, 3 (1978)

70. H.R. Lewis Jr., J. Math. Phys. 9, 1976 (1968)

71. M. Kruskal, J. Math. Phys. 3, 806 (1962)

72. P.G.L. Leach, Siam J. Appl. Math. 34, 496 (1978)

73. J.R. Ray, J.L. Reid, Phys. Lett. A 71, 317 (1979)

74. P.G.L. Leach, K. Andriopoulos, Appl. Anal. Discrete Math. 2, 146 (2008)

75. C. Rogers, C. Hoenselaers, J.R. Ray, J. Phys. A Math. Gen. 26, $2625(1993)$

76. W.K. Schief, C. Rogerts, A.P. Bassom, J. Phys. A Math. Gen. 29, 903 (1996)

77. P.G.L. Leach, J. Math. Phys. 21, 300 (1980)

78. S. Moyo, P.G.L. Leach, J. Math. Anal. Appl. 252, 840 (2000)

79. D. Bertacca, S. Matarrese, M. Pietroni, Mod. Phys. Lett. A 22, 2893 (2007)

80. D. Benisty, E.I. Guendelman, EPJC 77, 396 (2017)

81. D. Bertacca, A. Raccanelli, O.F. Piatella, D. Pietrobon, N. Bartolo, S. Matarrese, T. Giannantonio, JCAP 03, 039 (2011)

82. R. Brandenberger, J. Froehlich, R. Namba, JCAP 09, 069 (2019)

83. X. Dou, X.-H. Meng, Adv. Astron. 2011, 829340 (2011) 